\title{
The detection of intentional contingencies in simple animations in patients with delusions of persecution
}

\author{
S.-J. BLAKEMORE, ${ }^{1}$ Y. SARFATI, N. BAZIN AND J. DECETY \\ From the Institute of Cognitive Neuroscience, London; Department of Psychiatry, Centre Hôspitalier \\ de Versailles, Paris, France; and Center for Mind, Brain and Learning, University of Washington, \\ Seattle, WA, USA
}

\begin{abstract}
Background. It has been proposed that delusions of persecution are caused by the tendency to overattribute malevolent intentions to other people's actions. One aspect of intention attribution is detecting contingencies between an agent's actions and intentions. Here, we used simplified stimuli to test the hypothesis that patients with persecutory delusions over-attribute contingency to agents' movements.
\end{abstract}

Method. Short animations were presented to three groups of subjects: (1) schizophrenic patients; (2) patients with affective disorders; and (3) normal control subjects. Patients were divided on the basis of the presence or absence of delusions of persecution. Participants watched four types of film featuring two shapes. In half the films one shape's movement was contingent on the other shape. Contingency was either 'intentional': one shape moved when it 'saw' another shape; or 'mechanical': one shape was launched by the other shape. Subjects were asked to rate the strength of the relationship between the movement of the shapes.

Results. Normal control subjects and patients without delusions of persecution rated the relationship between the movement of the shapes as stronger in both mechanical and intentional contingent conditions than in non-contingent conditions. In contrast, there was no significant difference between the ratings of patients with delusions of persecution for the conditions in which movement was animate. Patients with delusions of persecution perceived contingency when there was none in the animate non-contingent condition.

Conclusions. The results suggest that delusions of persecution may be associated with the overattribution of contingency to the actions of agents.

\section{INTRODUCTION}

Persecutory delusions, in which a person holds a bizarre and paranoid belief with extraordinary conviction, despite experiences to the contrary and counter-arguments, are symptoms commonly associated with schizophrenia (Kraepelin, 1919; Ndetei \& Vadher, 1984; Johnstone, 1991), depression, bipolar disorder and schizoaffective disorder (Lattuada et al. 1999). Within the

\footnotetext{
1 Address for correspondence: Dr Sarah-Jayne Blakemore, Institute of Cognitive Neuroscience, 17 Queen Square, London WC1N 3AR.
}

cognitive approach to psychopathology it has been argued that processes involved in social inference, the processes by which we interpret the actions of other people and events involving others, play an important role in the development of paranoid delusions (Frith, 1992; Bentall et al. 2001). One such social inference process that may be associated with paranoid delusions involves inferring the causes of social interactions and events (Bentall et al. 2001). Bentall and colleagues have argued that paranoid beliefs may be a product of abnormal causal attributions (Bentall, 1994; Bentall et al. 2001). Indeed, patients with persecutory delusions 
over-attribute negative events to external causes (Kaney \& Bentall, 1989; Candido \& Romney, 1990; Fear et al. 1996) and to the actions of other people (Kinderman \& Bentall, 1997).

A second type of social inference process involves the attribution of intentions to other people's actions and behaviour. Understanding the intentional states of other people is an example of our inherent ability to understand other people's minds (referred to as having a 'theory of mind'; Premack \& Woodruff, 1978; Carruthers \& Smith, 1996). Frith (1992, 1994) has argued that a dysfunctional theory of mind may be implicated in psychotic symptoms including persecutory delusions. An impairment in understanding other people's intentions could lead to the belief that others have malevolent intentions, or that other people's actions are determined by the intention to harm the deluded person. Many studies have supported the theory that the understanding of intention is impaired in patients with paranoid delusions. Such patients are poorer at inferring intentions behind veiled speech acts, such as hints, than normal and psychiatric controls and than schizophrenic patients whose symptoms are in remission (Corcoran et al. 1995). A difficulty in answering questions that involve inferring mental states of a story character is specifically associated with persecutory delusions in schizophrenia (Frith \& Corcoran, 1996). Patients with paranoid delusions show impairments understanding jokes that require inferring the mental state of a character, but can understand 'physical' jokes that involve no mental state inference (Corcoran et al. 1997). Paranoid schizophrenic patients make significantly more mistakes on tasks that involve inferring the beliefs and intentions of the speaker in conversation (Tenyi et al. 2002). On the other hand, several studies have found that theory of mind impairments in psychotic patients are not specific to patients with persecutory delusions (Doody et al. 1998; Drury et al. 1998; Langdon \& Coltheart, 1999). Theory of mind impairment has been associated with formal thought disorder (Sarfati et al. 1997), thought and speech disorganization (Sarfati \& Hardy-Bayle, 1999) and psychomotor poverty (Mazza et al. 2001).

The tasks that have been used to test theory of mind in patients have generally employed complex stimuli and have involved relatively high-level theory of mind inferences, such as understanding cartoons or stories on the basis of a person's beliefs and desires. Much simpler stimuli such as videos of simple two dimensional shapes whose movement is 'animate,' that is self-propelled, lead normal control participants to attributions of mental states such as agency, intentions and emotions (Heider \& Simmel, 1944; Scholl \& Tremoulet, 2000). These videos, and other theory of mind stimuli, involve several types of 'agency' cue. One cue that an object is an agent and driven by intentions and desires is animate movement, that is movement that is self-propelled. A second agency cue is the presence of causal relationships between the animate movement of the shapes or story characters. An object that follows another object or reacts to its movement is perceived as being driven by internal intentions or goals. In the present study, our aim was to investigate the contribution of animate motion and causation at a distance to the detection of agency, and to test whether the perception of agency in simple animations is disturbed in patients with delusions of persecution.

We used computer-generated simple animations with two shapes that suggested neither 'body' nor 'face' nor any other biologically relevant morphological feature. These shapes had reduced behaviours: either linear motion, no motion or constant angular velocity rotations. All animations included only two objects in the roles of 'Prime Mover' and 'Reactive Mover' respectively. In each condition, the Prime Mover moved across the screen at constant speed. What was manipulated was its interaction with the Reactive Mover (Fig. 1, see Method for details). In some cases the movement of the Reactive Mover was a self-propelled (animate) rotation. When this was the case, the contingency between the two shapes' movement was manipulated by the exact timing of the Reactive Mover's rotation. In the animatecontingent condition the Reactive Mover made a self-propelled rotation when the Prime Mover was 'in view'. In the animate non-contingent condition the Reactive Mover's rotation occurred before the Prime Mover could be 'seen' through the window. In the rest of the stimuli no animate movement occurred. In these inanimate conditions, contingency between the shapes comprised a 'collision' between the two shapes. 


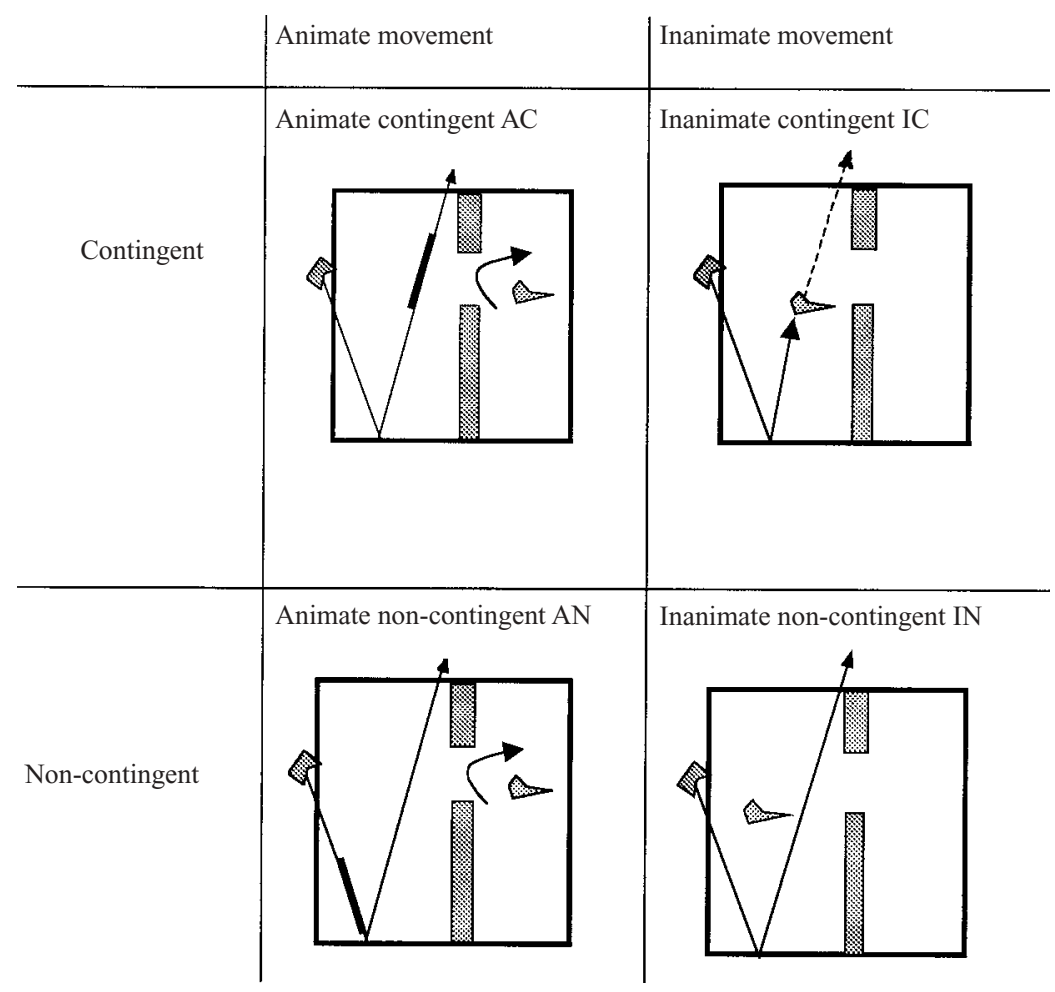

FIG. 1. Diagram illustrating the visual stimuli used in the experiment. In the animate contingent (AC) condition, the Reactive Mover rotates and appears to 'observe' the Prime Mover as it passes the window (indicated by the bold line). In the animate noncontingent (AN) condition the Reactive Mover rotates when the Prime Mover is 'out of sight'. In the inanimate contingent (IC) condition the Prime Mover collides with the Reactive Mover. In the inanimate non-contingent (IN) condition, the Prime Mover moves across the screen as in IC but moves past the Reactive Mover.

In the inanimate non-contingent stimuli no collision between the shapes occurred because the Reactive Mover was not in the path of the Prime Mover.

Participants were asked to state whether they thought the movement of one shape was in any way contingent on, or caused by, the movement of the other shape. They were also asked to rate the strength of the relationship between the movement of the two shapes in each condition. On the basis of the proposal that patients with delusions of persecution over-attribute causes to external events (Bentall et al. 2001) and are impaired at detecting other people's intentions (Frith, 1992), we predicted that such patients would be more likely to detect contingency between agents than normal control participants and patients without delusions of persecution. In contrast, there is no a priori reason to suppose that patients with delusions of persecution should show any abnormality in detecting contingency between inanimate objects (mechanical collisions).

\section{METHOD}

\section{Participants}

A group of 22 in-patients from the Department of Psychiatry at the Centre Hôspitalier de Versailles, France gave informed consent to participate in the current study. All patients were diagnosed by clinical interview using the ICD-10 (International Classification of Diseases; WHO, 1992).

The patients were divided into two groups according to the presence or absence of persecutory delusions, according to the PANSS interview (Kay et al. 1987) conducted by a psychiatrist (Y.S. or N.B.). The average ( \pm standard error) PANNS score on the persecutory delusions scale was $1 \cdot 5( \pm 0 \cdot 36)$ for the group of patients without persecutory delusions 
and 5.36 $( \pm 0 \cdot 23)$ for the group classified as experiencing persecutory delusions.

According to this classification a total of 12 (eight schizophrenic and four affective; two females; mean age $37 \cdot 4 \pm 5 \cdot 13$; years of education $7 \pm 0 \cdot 40$ ) patients had persecutory delusions. Ten (three schizophrenic and seven affective; three females; mean age $32 \cdot 1 \pm 2 \cdot 82$; years of education $8 \pm 0.65)$ patients were classified as not having these symptoms. A group of normal control participants $(N=14$; seven females; mean age $28.7 \pm 2.56$ years) also took part in this study. The normal control participants were younger and had spent a longer time in education $(17 \pm 0 \cdot 35$ years $)$ than the patients. There was no significant difference between the age $\left(t=1 \cdot 02_{19} ; P=0 \cdot 32\right)$ or between the number of years spent in education for the two patient groups based on data available for 17 patients $\left(t=-1 \cdot 20_{15} ; P=0 \cdot 25\right)$.

\section{Experimental design}

Stimuli from the following four conditions were visually presented to the participants.

In the animate contingent condition (AC), a 'Prime Mover' shape moves across the screen. A 'Reactive Mover' shape, which is positioned behind a window in a vertical wall, starts to rotate in the direction of the Prime Mover's motion at the moment when the Prime Mover moves past the window. The Reactive Mover stops moving when the Prime Mover has moved past the window and is 'out of view' (see Fig. 1, AC). This film was designed so that Reactive Mover appeared to move as a consequence of 'seeing' the Prime Mover.

In the animate non-contingent condition (AN) the Prime Mover moves across the screen, as in condition AC. The Reactive Mover rotates before the Prime Mover reaches the window when the Prime Mover is 'out of sight'. Thus, the movement of the Reactive Mover is not perceived as contingent on the movement of the Prime Mover (Fig. 1, AN).

In the inanimate contingent condition (IC), the Prime Mover moves across the screen and collides with the Reactive Mover, which is positioned in the path of the Prime Mover (Fig. 1, IC). The Reactive Mover moves off the screen. This condition was designed to appear as if the Prime Mover's movement caused (launched) the movement of the Reactive Mover.
In the inanimate non-contingent (IN) condition, the Prime Mover moves across the screen as in IC but moves past the Reactive Mover, which is positioned to the side of the path of the Prime Mover, so no contact is made between them (Fig. 1, IN).

Each stimulus image consisted of $512 \times 512$ pixels and 256 colours, lasted $4 \mathrm{~s}$ and the screen was updated at 15 frames/s. Stimuli were presented to the participants on a Macintosh Powerbook G4 using Quicktime. Each participant saw a total of 20 films comprising five different stimuli from each condition. The position of the Prime Mover's exit point (top, middle or bottom of the screen), the colour of the shapes (blue, green or red), the form of the shapes (spikedor smooth-edged) and the direction of motion (horizontal or vertical) were varied. The variation of these factors was counter-balanced between conditions. The order of stimulus presentation was pseudo-randomized and counterbalanced.

Before the experiment each participant was shown an exemplar stimulus from each of the four conditions, and was instructed to watch the movement of the two shapes in the visual displays. During the experiment each film was presented twice. After the first presentation of each film, participants were asked to watch the film again in order to respond 'yes' or 'no' to the question 'In your opinion, was there a relationship-direct or indirect-between the movement of the two shapes?' After the second presentation of the film, participants were also asked to rate the strength of the relationship between the movement of the two shapes on a scale from 0 to 10 , where 0 represented no relationship and 10 represented a very strong relationship. Participants were told that it was important to maintain the same scale all the way through the experiment and that the emphasis was on the relative values of their judgements, not the absolute values. For each of the four types of film, each participant was asked to describe what they perceived to be happening in the film.

\section{Control task}

In order to obtain an objective assessment of the ability of participants to rate a visual stimulus, and to follow the experimenter's instructions, participants were shown a further six stimuli 
(from the IN condition) and were asked to rate the height at which the Prime Mover shape exited the screen. The shape exited at the bottom left in two stimuli, the centre left in two stimuli and the top left of the screen in two stimuli. The stimuli were presented in a random order. Participants were asked to rate where on the screen the shape exited, on a scale from 0 to 10 , where 0 represented the very bottom of the screen and 10 represented the very top of the screen. Participants' contingency rating data were excluded from the analysis if their ordering in this objective control task was incorrect.

\section{Data analysis}

Analyses were performed on the data to test the hypothesis that the presence of delusions of persecution had a significant effect of the perception of contingency between animate objects. It was predicted that patients with delusions of persecution would perceive more contingency between the shapes whose movement was animate (agents) than patients without delusions of persecution and than normal control participants. In contrast, it was predicted that these patients' ratings of the strength of the relationship between the shapes in the IC and IN conditions would not significantly differ from the normal control participants' ratings. In other words, we predicted that there would be no difference in contingency ratings between subject groups for the inanimate stimuli, whereas there would be a significant difference between the contingency ratings of the patients with delusions of persecution and the other two subject groups for the animate stimuli.

Since each participant used their own subjective rating scale, the ratings need not conform to a normal distribution. Given the non-normal distribution of scores, Wilcoxon non-parametric matched pairs signed ranks test (Howell, 1997) was used to compare the ratings in the contingent versus the non-contingent conditions, within each participant group. Between group comparisons were made using a chi-squared test. Results were taken as significant if $P<0 \cdot 05$.

\section{RESULTS}

\section{Control trials}

All but one participant rated the exit position of the shape in the control task in the correct order. This participant had a diagnosis of schizophrenia and was classified as having persecutory delusions. His contingency rating data were excluded from further analysis.

\section{Contingency ratings analysis}

The results of the Wilcoxon non-parametric ranking test demonstrated that persecutory delusions had a significant effect on contingency ratings in the animate but not the inanimate conditions.

\section{Inanimate contingency ratings}

All three groups of participants showed a significant difference between their ratings for the IC and the IN conditions. The relationship between the shapes' movement in the IC condition was rated as significantly stronger than in the IN condition by the normal control participants (df $=13, \quad W=91, \quad P<0 \cdot 005$, two-tailed), the patients without persecutory delusions $(\mathrm{df}=9$, $\mathrm{W}=55, P<0 \cdot 01$, two-tailed), and the patients with persecutory delusions $(\mathrm{df}=10, \mathrm{~W}=66$, $P<0 \cdot 005$, two-tailed). When asked to describe what they saw in the inanimate contingent films, participants from all three groups reported that they perceived the Prime Mover to 'collide with' or 'smash into' and 'push' the Reactive Mover.

\section{Animate contingency ratings}

\section{Normal control participants}

The normal control participants rated the shapes' movement in the AC stimuli as significantly stronger than in the AN stimuli $(\mathrm{df}=13$, $\mathrm{W}=91, P<0.005$, two-tailed). When asked to describe what they saw, normal control participants reported that they perceived the Reactive Mover in the AC condition to be 'following' or 'watching' the Prime Mover. In the AN condition, normal control participants commented that the Reactive Mover 'moved of its own accord' and 'could not see' the Prime Mover.

\section{Patients without persecutory delusions}

The patients without persecutory delusions rated the relationship between the shapes as significantly stronger in the AC stimuli than in the AN stimuli ( $\mathrm{df}=9, \mathrm{~W}=55, P<0 \cdot 01$, twotailed). Like the normal control participants, when asked to describe what they saw, these patients tended to report that they perceived 
the Reactive Mover in the AC condition to be 'following' or 'watching' the Prime Mover. Patients without delusions of persecution commented that the Reactive Mover 'moved separately' and 'could not see' the Prime Mover.

\section{Patients with persecutory delusions}

There was no significant difference between the contingency ratings in the $\mathrm{AC}$ and $\mathrm{AN}$ conditions for patients with persecutory delusions (df $=10, W=5, P=0.409$, one-tailed). These patients reported that they perceived the Reactive Mover in the AC condition as 'following' or 'watching' the Prime Mover. Many participants in this group reported that the Reactive Mover 'heard', 'felt' or 'received magnetic information about' the Prime Mover in the AN condition.

\section{Comparison between groups}

We then calculated the difference between the contingency ratings for the contingent and noncontingent stimuli for both animate and inanimate conditions (AC-AN and IC-IN) for each participant. These mean difference ratings were used in the subsequent analysis. Because these scores were not appropriately distributed the differences between the groups in terms of the number of participants who showed the "normal' pattern of responding was examined using a chi-squared test. The number of participants showing the 'normal' response (a higher contingency rating for $\mathrm{AC}$ than $\mathrm{AN}$, and a higher contingency rating for IC than IN) was compared with the number of participants not showing this normal response in each participant group. Overall comparison of the contingency ratings in the animate conditions for the three groups revealed a significant difference between groups for the AC-AN difference ratings $\left(\chi^{2}(2)=12 \cdot 72, P<0 \cdot 01\right)$. Direct examination of the prediction showed that significantly fewer patients with delusions of persecution showed the normal response than patients without these symptoms $\left(\chi^{2}(1)=5.97, P<0.05\right)$. These results indicate that significantly fewer patients with delusions of persecution showed the normal response in the animate conditions $(\mathrm{AC}>\mathrm{AN})$ than patients without these symptoms and normal control participants, all of whom gave the AC condition a higher contingency rating than the AN condition (Fig. 2).

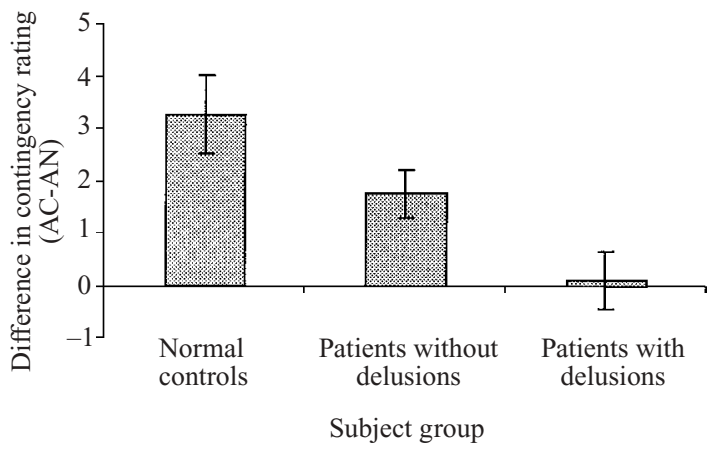

FIG. 2. Graph showing the mean ( \pm s.E.) difference in contingency ratings for the animate contingent $(\mathrm{AC})$ and animate non-contingent (AN) conditions for all three groups. The normal control subjects and patients without delusions of persecution rated the relationship between the shapes in the AC displays as significantly stronger than in the AN displays. There was no significant difference between the contingency ratings for the $\mathrm{AC}$ and $\mathrm{AN}$ displays for patients with delusions of persecution, hence the mean rating difference is close to zero.

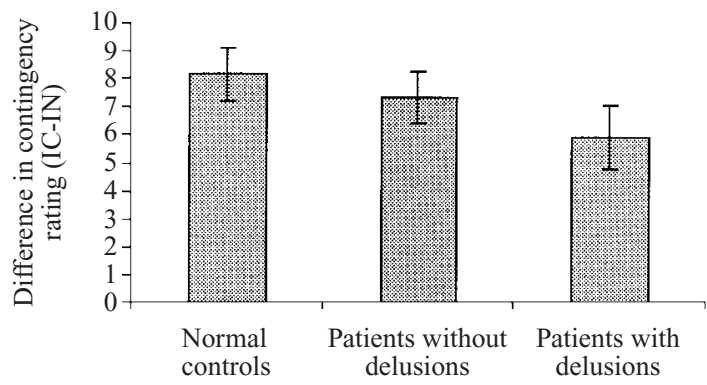

Subject group

FIG. 3. Graph showing the mean ( \pm s.E.) difference in contingency ratings for the inanimate contingent (IC) and inanimate noncontingent (IN) conditions for all three groups: normal control subjects, patients without delusions of persecution and patients with delusions of persecution. All three groups rated the relationship between the shapes in the IC displays as significantly stronger than in the IN displays.

For the inanimate stimuli, all participants from each group rated the IC stimuli as more contingent than the IN stimuli (Fig. 3).

\section{DISCUSSION}

The results of the current experiment demonstrate that patients with delusions of persecution are more likely than normal control subjects and patients without delusions of persecution to perceive contingency between the movement of shapes when the shapes move in an animate way, but not when their movement is purely 
mechanical. Although this study employed very simple visual stimuli that differed only in terms of highly constrained movement parameters, the results suggest that persecutory delusions might be characterised by an increased tendency to attribute contingency to other agents' behaviour.

It has been proposed that processes involved in social inference might play a role in the development of paranoid delusions (Frith, 1992; Bentall et al. 2001). Bentall and colleagues have suggested that paranoid beliefs may be a product of abnormal causal attributions to social interactions (Kaney \& Bentall, 1989; Bentall, 1994; Bentall et al. 2001). Frith has suggested that the inherent tendency to attribute intentions and other mental states to other people ('theory of mind') is abnormal in patients with delusions of persecution (Frith, 1992, 1994). Frith has argued that a dysfunctional theory of mind, including the understanding that other people's behaviour is driven by their intentions, desires and beliefs, may lead to the conviction that others have malevolent intentions directed towards the patient. There is empirical support for this theory (Corcoran et al. 1995, 1997; Frith \& Corcoran, 1996; Tenyi et al. 2002).

The tasks used to test theory of mind in patients have generally employed complex stimuli and involve relatively high-level inferences, such as understanding cartoons or stories on the basis of an person's beliefs and desires. These tasks implicitly involve several types of 'agency' cue, including the presence of biological characters, animate movement and the presence of causal relationships between actions of the shapes or story characters. In the present study, our aim was to disentangle these different aspects of agency detection by using very simple stimuli with tightly controlled psychophysics.

The current results lend support to the suggestion that patients with delusions of persecution show abnormal agency detection (Frith, 1992). Patients with delusions of persecution were more likely to detect contingency between the movement of animate shapes in displays in which normal control participants and patients without delusions of persecution perceived significantly less contingency (Fig. 2). When the Reactive Mover moved at the exact time the Prime Mover passed a window (condition AC; Fig. 1), subjects from all three groups perceived a contingent relationship between the two shapes, commenting that the Reactive Mover could 'see' and was 'following' the Prime Mover. In contrast, in the AN condition the Reactive Mover moved when the Prime Mover was 'out of sight'. In this condition normal control subjects and patients without delusions of persecution perceived the shapes as having a significantly less strong relationship than in the AC condition. Most subjects in these groups reported that the Reactive Mover 'could not see' the Prime Mover, and its movement was unrelated to the Prime Mover. In contrast, patients with delusions of persecution rated the relationship between the shapes as equally strong in both the $\mathrm{AC}$ and AN conditions. Many of these patients commented that the Reactive Mover 'heard', 'felt' or 'received magnetic information about' the Prime Mover, which caused it to rotate, in the AN condition. These patients therefore tended to attribute cause to the movement of the Reactive Mover in the films in which the Reactive Mover's movement was animate but in which the other two groups of subjects perceived significantly less contingency between the two shapes.

In contrast to their 'abnormal' pattern of response to the films containing animate movement, patients with delusions of persecution showed the 'normal' pattern of contingency ratings when the movement of the shapes was purely mechanical. All three groups rated the relationship between the shapes in the IC condition as significantly stronger than in the IN condition (Fig. 3). This demonstrates that the 'abnormal' contingency detection shown by the patients with delusions of persecution was specific to stimuli in which one shape's movement was animate. Animacy is one key cue to agency. The current results suggests that patients with delusions of persecution might over-attribute intentions and causes to the actions of agents. If this occurred in everyday life, it could be one explanation for their paranoid delusions, as suggested by Frith (1992).

Frith's theory proposes that delusions of persecution might be characterized by the belief that others have malevolent intentions, which are directed towards the patient. Our results do not bear on whether patients with delusions of persecution are more likely to perceive 
contingency between an agent's actions and his intentions towards the patient. In this study, subjects were asked to rate the relationship between two shapes in short animations; they did not make judgements about the shapes' actions in relation to themselves. From the current results it is difficult to assert whether the increased contingency rating in the $\mathrm{AN}$ condition in patients with delusions of persecution is a state or trait marker. The increased tendency to attribute contingency to animate movement might be considered a state marker because it is possible that some of the patients in the "without symptom' group would have at some stage experienced delusions of persecution. This might also account for contingency ratings of the patients without delusions of persecution, which showed a trend in the direction of the ratings for the patients with delusions of persecution (as can be seen in Fig. 2). It is not possible from this study to determine whether people with delusions of persecution at the time of the experiment would continue to show the same results when they are in remission. This is an important question that might be pursued in a future study.

Our results may have implications for the brain basis of delusions of persecution. Brain imaging studies that have employed tasks that involve inferring someone's intentions have been associated with activation of three key brain regions: the medial frontal cortex in the region of the paracingulate, the superior temporal sulcus and the temporal pole adjacent to the amygdala (for reviews see Frith \& Frith, 1999; Blakemore \& Decety, 2001; Frith, 2001). There is preliminary evidence that this network of brain areas responds abnormally to theory of mind tasks in schizophrenic patients (Brunet et al. 2003). Results from our lab indicate that a subset of these brain regions is involved when detecting contingency between animate shapes in films like those used in the current study (Blakemore et al. 2003). It would be interesting in the future to investigate whether these brain regions respond abnormally to these stimuli in patients with delusions of persecution.

S.J.B. is supported by a Wellcome Trust Research Fellowship. This research was supported by the Wellcome Trust UK, the Programme Cognitique from the Ministry of Education, France and INSERM, France.

\section{REFERENCES}

Bentall, R. P. (1994). Cognitive biases and abnormal beliefs : towards a model of persecutory delusions. In The Neuropsychology of Schizophrenia (ed. A. S. David and J. Cutting), pp. 337-360. Lawrence Erlbaum: London.

Bentall, R. P., Corcoran, R., Howard, R., Blackwood, N. \& Kinderman, P. (2001). Persecutory delusions: a review and theoretical integration. Clinical Psychology Review 21, 1143-1192.

Blakemore, S.-J. \& Decety, J. (2001). From the perception of action to the understanding of intention. Nature Reviews Neuroscience 2 , 561-567.

Blakemore, S.-J., Boyer, P., Pachot-Clouard, M., Meltzoff, A., Segebarth, C. \& Decety, J. (2003). The detection of contingency and animacy from simple animations in the human brain. Cerebral Cortex (in the press).

Brunet, E., Sarfati, Y., Hardy-Baylé, M. C. \& Decety, J. (2003). Abnormalities of brain function during a nonverbal theory of mind task in schizophrenia. Neuropsychologia 41, 1574-1582.

Candido, C. L. \& Romney, D. M. (1990). Attributional style in paranoid vs. depressed patients. British Journal of Medical Psychology 63, 355-363.

Carruthers, P. \& Smith, P. K. (1996). Theories of Theories of Mind. Cambridge University Press: Cambridge.

Corcoran, R., Mercer, G. \& Frith, C. D. (1995). Schizophrenia, symptomatology and social inference: investigating 'theory of mind' in people with schizophrenia. Schizophrenia Research 17, 5-13.

Corcoran, R., Cahill, C. \& Frith, C. D. (1997). The appreciation of visual jokes in people with schizophrenia: a study of 'mentalizing' ability. Schizophrenia Research 24, 319-327.

Doody, G. A., Gotz, M., Johnstone, E. C., Frith, C. D. \& Owens, D. G. (1998). Theory of mind and psychoses. Psychological Medicine 28, 397-405.

Drury, V. M., Robinson, E. J. \& Birchwood, M. (1998). 'Theory of mind' skills during an acute episode of psychosis and following recovery. Psychological Medicine 28, 1101-1112.

Fear, C., Sharp, H. \& Healy, D. (1996). Cognitive processes in delusional disorders. British Journal of Psychiatry 168, 61-67.

Frith, C. D. (1992). The Cognitive Neuropsychology of Schizophrenia. Lawrence Erlbaum Associates: Hove.

Frith, C. (1994). Theory of mind in schizophrenia. In The Neuropsychology of Schizophrenia (ed A. S. David and J. C. Cutting), pp. 147-161. Erlbaum: Hove.

Frith, C. D. \& Corcoran, R. (1996). Exploring 'theory of mind' in people with schizophrenia. Psychological Medicine 26, 521-530.

Frith, C. D. \& Frith, U. (1999). Interacting minds - a biological basis. Science 286, 1692-1695.

Frith, U. (2001). Mind blindness and the brain in autism. Neuron 32 969-979.

Heider, F. \& Simmel, M. (1944). An experimental study of apparent behavior. American Journal of Psychology 57, 243-249.

Howell, D. C. (1997). Statistical Methods for Psychology, 4th edn. Duxbury Press: London.

Johnstone, E. C. (1991). Defining characteristics of schizophrenia. British Journal of Psychiatry (suppl. 13), 5-6.

Kaney, S. \& Bentall, R. P. (1989). Persecutory delusions and attributional style. British Journal of Medical Psychology 62, 191-198.

Kay, S., Fiszbein, A. \& Opler, L. (1987). The Positive and Negative Symptom Scale for schizophrenia. Schizophrenia Bulletin 13, 261-276.

Kinderman, P. \& Bentall, R. P. (1997). Causal attributions in paranoia and depression: internal, personal, and situational attributions for negative events. Journal of Abnormal Psychology 106, 341-345.

Kraepelin, E. (1919). Dementia Praecox and Paraphrenia. Livingstone: Edinburgh.

Langdon, R. \& Coltheart, M. (1999). Mentalising, schizotypy, and schizophrenia. Cognition 71, 43-71.

Lattuada, E., Serretti, A., Cusin, C., Gasperini, M. \& Smeraldi, E. (1999). Symptomatologic analysis of psychotic and non-psychotic depression. Journal of Affective Disorders 54, 183-187. 
Mazza, M., De Risio, A., Surian, L., Roncone, R. \& Casacchia, M. (2001). Selective impairments of theory of mind in people with schizophrenia. Schizophrenia Research 47, 299-308.

Ndetei, D. M. \& Vadher, A. (1984). Frequency and clinical significance of delusions across cultures. Acta Psychiatry Scandinavia 70, 73-76.

Premack, D. \& Woodruff, G. (1978). Does the chimpanzee have a theory of mind? Behavioural and Brain Sciences 4, 515-526.

Sarfati, Y. \& Hardy-Baylé, M. C. (1999). How do people with schizophrenia explain the behaviour of others? A study of theory of mind and its relationship to thought and speech disorganization in schizophrenia. Psychological Medicine 29, 613-620.
Sarfati, Y., Hardy-Baylé, M. C., Besche, C. \& Widlocher, D. (1997). Attribution of intentions to others in people with schizophrenia: a non-verbal exploration with comic strips. Schizophrenia Research 25, 199-209.

Scholl, B. J. \& Tremoulet, P. D. (2000). Perceptual causality and animacy. Trends in Cognitive Science 4, 299-309.

Tenyi, T., Herold, R., Szili, I. M. \& Trixler, M. (2002). Schizophrenics show a failure in the decoding of violations of conversational implicatures. Psychopathology 35, 25-27.

World Health Organization (1992). International Classification of Diseases-10 (ICD-10) Classification of Mental and Behavioural Disorders. WHO: Geneva. 